\title{
Un breve consejo médico puede ser efectivo en bebedores de alcohol
}

Fleming MF, Barry KL, Manwell LB et al. Brief physician advice for problem alcohol drinkers.

JAMA 1997; 277:1039-1045

\section{Objetivo}

Evaluar la eficacia del consejo médico para disminuir el consumo de alcohol y la utilización de servicios de salud en los bebedores.

\section{Diseño}

Ensayo clínico doble ciego, randomizado y controlado. Seguimiento a un año.

\section{Lugar}

Se reclutaron 64 médicos de familia e internistas generales de distintos centros urbanos y rurales de Wisconsin, E.E.U.U.

\section{Pacientes}

Los pacientes fueron seleccionados de la población de los 64 médicos de acuerdo a un cuestionario acerca de su salud, al consentimiento correspondiente y entrevistas personales con uno de los médicos. Incluyeron hombres que consumían más de 14 copas semanales y mujeres que consumían más de 11. Fueron excluidos aquellos pacientes que habían tenido un tratamiento de abstinencia en el último año, tomaran más de 50 copas por semana o reportaran intento de suicidio.

\section{Intervención}

A cada paciente se le suministró información escrita y recomendaciones generales sobre su salud y estilo de vida general. A los pacientes del grupo intervención se les suministró material y consejo médico específico dirigido al problema del alcohol.

\section{Medición de Resultados}

Las variables de medición fueron: cambios en el uso de alcohol, días de internación, e indicadores de utilización de recursos (consultas a los centros de atención). El seguimiento se realizó en forma telefónica. Para evaluar la eficacia de la intervención se interrogó telefónicamente también a los miembros de la familia.

\section{Resultados}

Se incluyeron 392 pacientes ( $62 \%$ hombres) en el grupo tratamiento (GT) y 382 pacientes (62\% hombres) en el grupo control (GC).

\begin{tabular}{llllll}
\multicolumn{2}{c}{ Basal } & \multicolumn{2}{c}{ Al año } & \multicolumn{2}{c}{ reducción al año } \\
GT & GC & GT & GC & GT & GC \\
19.1 & 18.9 & 11.5 & 15.5 & $40 \%$ & $18 \%$ \\
5.6 & 5.3 & 3.1 & 4.2 & $46 \%$ & $21 \%$ \\
160 & 176 & 60 & 119 & $62 \%$ & $32 \%$
\end{tabular}

a. Copas ingeridas en los últimos 7 día

b. Episodios compulsivos de ingesta alcohólica

c. Bebida excesiva*

Bebida excesiva fue definido como la ingesta de más de 20 copas por semana para los hombres y 13 para las mujeres.

Quienes recibieron la intervención tuvieron un Odds Ratio (OR) de 2.15 (IC 95\%:1.58-2.93) de reducir un $20 \%$ más el consumo de alcohol. Hubo diferencias en la cantidad de días de hospitalización (314 GC vs 178 GE). En el análisis multivariado la exposición a la intervención del médico fue la única variable significativa. Las demás (sexo, tabaquismo, edad, depresión, desorden de la conducta) no se asociaron significativamente al éxito.

\section{Conclusiones}

La intervención breve tuvo un efecto beneficioso en disminuir el consumo de alcohol en pacientes bebedores moderados.

\section{Comentario}

El presente trabajo muestra que una intervención breve en los pacientes alcohólicos moderados puede ser de gran utilidad (los autores consideraron como resultado exitoso a una reducción de por lo menos un $20 \%$ en la ingesta de bebidas alcohólicas).

Las limitaciones del estudio se basan fundamentalmente en la utilización de los auto-cuestionarios para una enfermedad como el alcoholismo donde puede haber subregis- tros. Además si hubo subregistro este se distribuiría de igual manera en los dos grupos ya que es un estudio randomizado. En cuanto al número de días de internación no se tuvo control de posibles internaciones en otros centros de salud. En este tipo de trabajo puede haber un efecto llamado "complacer al médico" donde los pacientes pueden reportar efectos beneficiosos inexistentes de la intervención. Para evitar este sesgo fue importante haber entrevistado telefónicamente a los familiares. En resumen, es importante que todo médico de atención primaria interrogue acerca del consumo de alcohol pues nuestro consejo ha demostrado ser una valiosa herramienta.

Dr. Alberto Velazquez Unidad de Medicina Familiar y Preventiva Hospital Italiano de Buenos Aires 